\title{
LA OBRA MARTIANA: SU COMPRENSIÓN EN LAS CLASES ESPAÑOL-LITERATURA EN 7MO GRADO
}

\author{
Maité Felicia Valdivia Valdés ${ }^{[1]}$ \\ Dianelys Rigo Prado ${ }^{[2]}$ \\ Kira María Larrea Hidalgo ${ }^{[3]}$
}

\section{Resumen}

Nadie ha dudado de la funcionalidad de la lectura para el dominio del propio lenguaje y para el manejo de información, así como para adquirir dignidad, sabiduría desde una praxis del "pensar por sí propio, el ejercicio íntegro de sí y el respeto íntegro a los demás". Por eso saber leer los diferentes textos de la obra martiana y comprenderlas, constituye un reto en cuanto a valorar la vida y obra de humanismo y estética de José Martí, paradigma en la trascendencia humana. A esto obedece el interés en "La obra martiana: su compresión en las clases de Español-Literatura". El tipo de investigación es cualitativa con nivel correlacional, fue aplicada en un grupo de 7 mo grado de la enseñanza Secundaria "Mártires de La Sierpe", la muestra se seleccionó de forma intencional, 20 estudiantes de 7 mo grado. El aporte ha consistido en sistematizar actividades que desarrollen habilidades de compresión textual y una mayor motivación por parte de los estudiantes hacia la obra de José Martí y promover el diálogo abierto hacia su valoración y acercamiento a lo íntegro en el acontecer de la libertad y las autonomías fundantes.

Palabras clave: lectura; lenguaje; pensar por sí propio; obra martiana; compresión; Español-Literatura.

\section{Summary}

No one has doubted the functionality of reading for the dominion o language itself and to manage information, as well as to acquire dignity and wisdom from a praxis of "thinking for yourself, the full exercise of yourself and the full respect for others". So to read different texts of Marti's work and understand it, is a challenge in terms of valuing life, humanist work and esthetics of Jose Marti, paradigm in human importance. This reflects our interest in "Marti's work: Its comprehension in SpanishLiterature classes."

[1] Universidad “José Martí Pérez” Sancti Spíritus, Cuba. Correo electrónico: maite@uniss.edu.cu. Calle 5ta: Edificio 14-a Apartamento 6 entre calle Tercera y calle Novena La Sierpe Sancti-Spíritus.

[2] Universidad “José Martí Pérez” Sancti Spíritus, Cuba. Correo electrónico: dianelys@uniss.edu.cu Calle A, casa nro 9 Zona Norte, La Sierpe Sancti-Spíritus.

[3] Máster en Ciencias de la Educación y en Desarrollo Local. Profesora de la Universidad " José Martí Pérez". Correo. kira@uniss. edu.cu 
The research is qualitative with correlational level, and it was applied to a group of 7 th grade of secondary school by the name of "Martires de la Sierpe"; the sample was selected intentionally, 20 students from 7 th grade. The contribution has been to systematize activities that develop skills of textual comprehension and a higher motivation of students to the work of Jose Marti, as well as to promote open dialogue to its appreciation.

Keywords: Reading; language; think for yourself; Marti's work; comprehension; Spanish-Literature.

\section{Introducción}

En Cuba, nace el poeta y libertador José Martí. A la libertad e independencia de su patria consagró su vida y por ella murió: por nuestra América. Martí nace con vocación y forja su luz para educar. Una inclinación irrefrenable lo impulsó al aula y a la redacción de textos literarios para niños, pues toda su vida la dedicó a sembrar consejos, amor e inspiración en su amplia obra escrita y a buscar caminos en el arte de educar.

Leer, escribir, contar: eso es todo lo que les parece que los niños necesitan saber. Pero: ¿A qué leer, si no se les infiltra la afición a la lectura, la convicción de que es sabrosa y útil, el goce de ir levantando el alma con la armonía y grandeza de conocimientos? ¿A qué escribir, si no se nutre la mente de ideas, ni se aviva el gusto de ellas? ¡Qué no se sabe bien sino lo que se descubre! (José Martí).

Esta concepción traza pautas en el estudiante como agente activo de su propio aprendizaje. Con voluntad y dedicación en la compresión de sus textos se puede aprender y descubrir exitosas posibilidades inimaginables, una gran expresividad y el contacto con la imaginación fabulosa. Se considera necesario estudiar a Martí en cada texto de su a obra ¡Hay que leer y estudiar al maestro! Por ser un escritor de todos los tiempos y universal. Leer es informarse y formarse es desarrollar el lenguaje y con él la hondura y las perspectivas de horizontes del pensamiento. Es ampliar la capacidad de la razón y del juicio, del conocimiento teórico y del aumento de posibilidades de la aplicación de esto a la práctica.

En las últimas décadas las concepciones acerca de la comprensión lectora ha suscitado una gran problemática que parte de los diferentes enfoques y criterios al abordar los problemas teóricos que deben tener en cuenta para su definición y esto tiene gran implicación en lo que respecta a su enseñanza y evaluación. Es por eso que autores de diferentes latitudes se refieren a sus obras a la necesidad de proporcionar a los estudiantes los conocimientos y habilidades que le permitan la interpretación de diferentes tipos de textos, a pesar de ello, actualmente los estudiantes presentan insuficiencia en la comprensión que influye en el aprendizaje de las diferentes 
asignaturas. "La lectura es un acto de crear y recrear ideas, no de consumirlas pasivamente como un depósito."

Es evidente que la actitud intelectual de la escuela debe realizarse sobre la base de una fuerte motivación de los estudiantes hacia la lectura. La preocupación porque las personas lean se acentúan cada vez más; no cabe duda de que la lectura es un instrumento básico de adquisición de conocimientos cuando se realiza de forma activa. Por la importancia que reviste el proceso lector se ha investigado esta temática, lo que a provocado que el concepto de lectura haya evolucionado hasta acomodarse a la necesidad de los cambios de la vida moderna. El estudiante cotidiano ha de emplear el idioma vinculado con el pensamiento, lo necesita para expresarse cada vez mejor. La lectura debe dar una preparación a los estudiantes, contribuir al desarrollo de habilidades e inculcar el amor al estudio. El cumplimiento de enseñar la lectura tiene un carácter integrador, es decir, la unidad indisoluble entre sus componentes: Lectura, expresión oral y escrita, ortografía, gramática y caligrafía. Para lograr todo esto es imprescindible una buena orientación metodológica, teniendo como premisa fundamental la integración de los componentes de la asignatura, de manera que puedan obtener, evaluar y utilizar la información que les brindan los textos de diversas naturalezas con los que se ponen en contacto. Con la lectura se dirige la atención del estudiante hacia el dominio de la técnica y unidad con la ciencia y las artes de la civilidad, a esto se trabaja.

\section{Materiales y métodos}

\section{Procedimiento Metodológico}

1.1 Los métodos teóricos se utilizaron para la interpretación conceptual de los datos empíricos encontrados, y al elaborar la propuesta práctica, posibilita explicar los hechos y profundizar en las relaciones esenciales y cualidades fundamentales de los procesos no observables directamente; formular las principales regularidades del funcionamiento y desarrollo de los procesos, mediante estos métodos y procedimientos se conforma el marco teórico del trabajo. Los métodos empíricos se emplearon fundamentalmente en la etapa del diagnóstico, en la acumulación empírica y en la comprobación experimental de la hipótesis de la investigación.

1,2 Los métodos del nivel estadístico o matemático permitieron colectar, organizar, resumir, presentar y analizar datos relativos del objeto del estudio.

1.3 El presente trabajo de investigación cualitativa con un nivel de profundidad correlacional, se realizó con la siguiente población y muestra. La población está formada por los 93 estudiantes correspondiente al total de la matricula general de 7 mo grado de la secundaria "Mártires de La Sierpe" del municipio La Sierpe provincia Sancti-Spíritus, situada en el Consejo Popular La Sierpe. 
La muestra, la integran 20 estudiantes del grado 7 mo grupo 1, que representa el $100 \%$ de la matrícula y el 21,5\% de la matrícula general del grado.

\section{Resultados}

\section{Resultados del diagnóstico actual}

Los resultados alcanzados en el diagnóstico inicial evidencian que los escolares investigados presentan insuficiencias en el desarrollo de habilidades de comprensión. La propuesta consiste en actividades en las cuales se pretende potenciar el desarrollo de habilidades de comprensión en los estudiantes de 7 mo Grado. Por cuanto se fundamenta a partir de la teoría acerca de la unidad de la conciencia y la actividad, donde al criticar la comprensión idealista de la conciencia Carlos Marx, Federico Engels y Vladimir I. Lenin mostraron convincentemente que no sólo en el proceso histórico, sino en el ontogénico resulta imposible considerar el problema de la fundación de la conciencia y el establecimiento de la personalidad independiente de la relación de esta con la actividad. En el proceso de la actividad conjunta con sus coetáneos, el estudiante aprende a comprender a los demás, a comprenderse, a dirigirse y a valorar sus actos.

Al elaborar la propuesta se tuvo en cuenta las características de los estudiantes, analizando su manera de actuar, sus conocimientos adquiridos en grados inferiores, así como la creación de condiciones necesarias, relación de métodos de dirección y procedimientos adecuados; además del corte pedagógico y el nivel de creatividad para poder propiciar la motivación e interés del estudiante hacia la actividad y así lograr el éxito de contenido que a través de esto se quiso llegar.

Las actividades se corresponden con los objetivos y contenidos de la asignatura Español-Literatura de 7 mo grado, así como las habilidades que pretendan desarrollar. Estas pueden ser aplicadas en cualquier grupo de estudiantes del grado, pues fueron elaboradas considerando las características psicológicas de los mismos en esta edad. Las actividades que conforman la propuesta alcanzan un total de 15, las mismas se proponen por su forma de ejecución, procedimientos y medios a emplear, para las mismas se utilizan los diferentes niveles cognitivos con distractores a partir de los diferentes tipos de textos de la obra martiana. Todas ellas están en función del desarrollo de habilidades de comprensión.

Las mismas están dirigidas a los estudiantes para ampliar su horizonte creativo y estimular la potenciación de las actividades, el desarrollo de habilidades de comprensión: son creativas, novedosas, motivadoras e interesantes que le permiten al estudiante convertirse en agente de su propio aprendizaje y captar todos los conocimientos expuestos en la vigente e infinita obra martiana. 


\section{HUMANIDADES}

Estas son razones suficientes por las cuales estas actividades se basan en la obra martiana en la cual encontramos como ideal al hombre, aquel que fue generoso y creador. De su obra se desprenden valores como amor a la patria, amor al trabajo, a la solidaridad humana; pero, resulta necesario acercar este paradigma a la propia vida del niño, no basta con que conozcan las cualidades que caracterizan a José Martí, y que sean capaces de descubrirlos en otras personas y en ellos mismos.

\section{Conclusiones}

La propuesta de actividades aplicadas contribuyó en gran medida a resolver la problemática existente, logrando que los estudiantes desarrollen habilidades de comprensión en las asignaturas con los diferentes texto del ideario martiano, lo que se evidencia en la comparación de los resultados de las constataciones efectuadas en el presente trabajo demostrándose así la veracidad de la hipótesis.

\section{Recomendaciones}

1. Valorar en la preparación metodológica de la Secundaria "Mártires de La Sierpe", la posibilidad de aplicar en otras aulas de 7 mo grado la experiencia, con las adecuaciones correspondientes.

2. Continuar enriqueciendo el tema abordado en este trabajo a fin de mejorar las actividades que permitan un aprovechamiento mejor de la propuesta.

\section{Lista de referencia}

Álvarez Valdés, Pedro Luis (1997). La aplicación de estrategias: una vía para la Comprensión lectora en 7. grado. En Tesis (Opción al grado académico de Máster en Didáctica del Español y la Literatura) Instituto Superior Pedagógico Enrique José Varona. Ciudad de La Habana.

Arias Leiva, Georgina (S/f). Horadar al texto. La comprensión del texto en la enseñanza primaria, Material impreso.

Cairney Trevor, H. (1996). Enseñanza de la comprensión lectora. Ministerio de Educación y Ciencia, Madrid.

Carbonel de Grampone; María A. (1989). ¿Qué es leer? y otras precisiones para Maestros inexpertos, en Revista Lectura y Vida, año 10, No.3. Santiago de Chile.

Dubois, María Eugenia (1986). Interrogantes sobre comprensión lectora, en RIDE - CAB, año 6, No. 14, Lima. 
Espinosa Martínez, Idolidia (1999). Estrategias de enseñanza - aprendizaje para la Comprensión del texto científico en quinto grado. En Tesis (Opción al título académico de Máster en Educación). Instituto Superior Pedagógico Cap. Silverio Blanco Núñez, Sancti Spíritus.

García Alzola, Ernesto (1992). Lengua y Literatura. Editorial Pueblo y Educación, La Habana.

García Pers, Delfina (1978). Didáctica del idioma españos I parte. Editorial Pueblo y Educación, La Habana.

Grass Gallo, Elida y Nayiri Fonseca (1992). Técnicas básicas de lecturas. Editorial Pueblo y Educación, La Habana.

Jiménez Rivero, Mayte y Aileen Díaz Bernal (2005). Folleto de Lengua Española 6. Grado. Instituto Central de Ciencias Pedagógicas.

León Cascón, José A y Juan A. García Madruga. Comprensión de textos e Instrucción. En fotocopia No. 102 CDIP ISP Sancti Spíritus.

Mañalich Suárez, Rosario y otros (1999). Taller de la palabra. Editorial Pueblo y Educación, La Habana.

Martín Santamaría, Nemesio. La función de la memoria en la comprensión Lectora. En fotocopia N. 96 CDIP Sancti Espíritus.

Martínez Suárez, Marta (1997). Estrategias que facilitan la comprensión y su motivación hacia la lectura hacia la lectura en gno. grado. En Tesis (Opción al grado académico de Máster en Didáctica del Español - Literatura) Instituto Superior Pedagógico Enrique José Varona.

Meza Cuevas, Luis Eduardo (1990). Educación para la lectura. En Reflexiones. Revista de la Facultad de Educación. Universidad Autónoma de Buramanga Vol. 3 N. pp. $15-21$.

MINED. (1989). Programas, Libro de texto y OM de 6. grado. Editorial Pueblo y Educación, La Habana.

Mined. Seminario Nacional para educadores en sus siete ediciones.

Montaño Calcines, Juan Ramón (S/f). Tras las huellas del texto. Una reflexión sobre La lectura. pp 61-64. 


\section{HUMANIDADES}

Rodríguez Pérez (2004). Editorial Pueblo y Educación, La Habana.

Morles, Armando (1985). Entrenamiento en el uso de estrategias para comprender la Lectura. En la Educación. Revista Interamericana de Desarrollo Educacional N.98 Whashington. pp. $79-49$.

Pichardo, Hortensia (1990). Lectura para niños. Editorial Pueblo y Educación, Ciudad de La Habana.

Santo Canet, Monserrat (1994). Animación a la lectura. En Vela Mayor. Revista de Anaya. Educación. Año 1 N. 1 Madrid. pp. 13 - 19.

Van Dijk, T. (1983). La ciencia del texto. Ediciones Paidós, Barcelona.

Velásquez Delgado, Ana Margarita (S/f). La comprensión de textos: una estrategia de Enseñanza - aprendizaje basada en el texto periodístico, en CDIP ISP Cap. Silverio Blanco Núñez S. Spíritus.

Vigotsky, Leo S. (1981). Pensamiento y Lenguaje. Edición Revolucionaria, Ciudad de La Habana.

Vitier, Cintio (1995). Cuadernos Martianos I Editorial Pueblo y Educación. Ciudad de La Habana.

Vitier, Cintio y Fina García Marruz (1995). Guía para los maestros de las "Aulas Martianas". Editorial Pueblo y Educación. pp.1-6. 